\title{
Estrategias para la enseñanza del pensamiento computacional y uso efectivo de tecnologías en educación infantil: una propuesta inclusiva
}

\author{
Strategies for teaching computational thinking and effective use of \\ technologies in childhood education: an inclusive proposal
}

\author{
Carina Soledad González-González \\ Universidad de La Laguna / Universidad Oberta de Catalunya (España) \\ carina.gonzalez@ieee.org
}

Recibido: 1/12/2019

Aceptado: 9/12/2019

Publicado: 26/12/2019

\section{RESUMEN}

En los últimos años, ha habido un impulso para introducir la enseñanza de la programación y el pensamiento computacional en la educación, y la robótica es una excelente herramienta para lograr esto. Sin embargo, la integración de estas habilidades fundamentales en los planes de estudio formales y oficiales sigue siendo un desafío y los educadores necesitan perspectivas pedagógicas para integrar adecuadamente los conceptos de robótica, programación y pensamiento computacional en sus aulas. Por lo tanto, en éste artículo se presenta una propuesta metodológica basada en los principios del marco de Desarrollo Tecnológico Positivo (PTD), el movimiento Maker, el constructivismo, la educación inclusiva y el aprendizaje a través de juegos, especialmente diseñado para la educación infantil. Esta propuesta ha sido validada en diferentes contextos mostrando la efectividad de la misma.

\section{PALABRAS CLAVE}

Ciencias tecnológicas; Método educativo; Enseñanza y formación.

\begin{abstract}
In recent years, there has been an impulse to introduce the teaching of programming and computational thinking in education, and robotics is an excellent tool to achieve this. However, the integration of these fundamental skills into formal and official curricula remains a challenge and educators need pedagogical perspectives to properly integrate the concepts of robotics, programming and computational thinking in their classrooms. Therefore, this article presents a methodological proposal based on the principles of the framework of Positive Technological Development (PTD), the Maker movement, constructivism, inclusive education and playful learning, specially designed for early childhood education. This proposal has been validated in different contexts showing its effectiveness.
\end{abstract}

\section{KEYWORDS}

Technological sciences; Educational method; Teaching and training. 


\section{CITA RECOMENDADA}

González-González, C. S. (2019). Estrategias para la enseñanza del pensamiento computacional y uso efectivo de tecnologías en educación infantil: una propuesta inclusiva. RIITE. Revista Interuniversitaria de Investigación en Tecnología Educativa, 7, 85-97. Doi: http://dx.doi.org/10.6018/riite.405171

\section{Principales aportaciones del artículo y futuras líneas de investigación:}

- Método educativo inclusivo para la enseñanza de la programación y el pensamiento computacional

- Conjunto de principios educativos para la enseñanza de la programación

- Propuesta de ideas poderosas y objetivos de aprendizaje a trabajar en la enseñanza de la programación y pensamiento computacional en la educación infantil

\section{INTRODUCCIÓN}

Los niños y niñas de todo el mundo están siendo criados en entornos saturados de dispositivos inteligentes. Al mismo tiempo, existe una creciente necesidad de una fuerza laboral futura que comprenda la tecnología, dada esta nueva realidad, los programas educativos nacionales y las iniciativas privadas se están centrando en la alfabetización STEM (Ciencia, Tecnología, Ingeniería y Matemáticas), haciendo de la codificación/programación y el pensamiento computacional una prioridad para la educación (Manches y Plowman, 2015). Sin embargo, la investigación ha encontrado que las intervenciones educativas en la educación infantil están relacionadas con costos más bajos y efectos más duraderos que las intervenciones que comienzan más tarde (Cunha y Heckman, 2007). Además, algunos estudios demuestran estereotipos basados en el género que involucran carreras STEM (Metz, 2007; Steele, 1998) y menos obstáculos para ingresar a la fuerza laboral (Madill et al., 2007; Markert, 1996) cuando los niños/as están expuestos a STEM en la infancia (Metz, 2007; Steele, 1998).

Por otra parte, diferentes estudios han demostrado el potencial de la educación en robótica en los primeros años (Jung y Won, 2018). Algunos de ellos han presentado métodos para implementar un plan de estudios robótico (Bers, 2010), para evaluar las habilidades de pensamiento computacional (Roman-González, Moreno-León y Robles, 2019; Siu-Cheung, 2019; Chen et al., 2017), para desarrollar funciones ejecutivas (Di Lieto et al., 2017), actitudes hacia la sociedad y la ciencia (Kandlhofer y Steinbauer, 2016) y las características tecnológicas de los robots y las interacciones (Burlson et al., 2017; Serholt, 2018). Sin embargo, la investigación sobre robótica y pensamiento computacional en la educación infantil aún se encuentra en sus primeras etapas (Öztürk y Calingasan, 2018; Ching, Hsu y Baldwin, 2018; Chen et al., 2017; García-Peñalvo y Mendes, 2018). Varios estudios se han centrado en aspectos tecnológicos o de interacción con la tecnología o en currículos de robótica, en lugar de como el alumnado se involucra y aprende y cómo los docentes introducen las nuevas habilidades en sus aulas y currículos (Jung y Won, 2018; Serholt, 2018).

Por ello, a través de la realización de una estancia de investigación con un grupo experto en educación infantil y robótica liderado por la profesora Marina Umaschi Bers, se estudió el modelo pedagógico-tecnológico desarrollado por el grupo de investigación "DevTech". Concretamente, los objetivos que se persiguieron fueron: a) diseñar un programa formativo dirigido al profesorado de Educación Infantil que permita dotarles de la metodología y herramientas que puedan utilizar en el aula para el desarrollo de diferentes actividades que ayuden al aprendizaje, por parte del alumnado, de las diferentes materias que componen este nivel educativo; b) investigar las tecnologías, las metodologías, las estrategias y en las tendencias actuales de enseñanza-aprendizaje del pensamiento computacional y c) proponer mejoras y adaptaciones en las metodologías para una enseñanza inclusiva del pensamiento computacional. En este artículo se centrará en describir este último objetivo, realizando una 
propuesta metodológica realizada para la enseñanza inclusiva de la programación y el pensamiento computacional para la educación infantil.

\section{ESTRATEGIAS PARA LA ENSEÑANZA DEL PENSAMIENTO COMPUTACIONAL Y LA PROGRAMACIÓN: UNA PROPUESTA INCLUSIVA}

En esta sección se describirán los principios pedagógicos y las principales estrategias de una propuesta inclusiva de enseñanza del pensamiento computacional y la programación para la educación. Dicha propuesta ha sido validada en diferentes estudios realizados y descritos en (Bers, González y Armas, 2019; González-González et al., 2019; González, Cáceres y Violant, 2019).

\subsection{Fundamentos y principios}

Después de estudiar las infraestructuras tecnológicas y metodologías empleadas en laboratorios de robótica infantil, el diseño de entorno que se propone en éste trabajo se basa en el movimiento de Maker (Halverson y Sheridan, 2014) y los principios pedagógicos del Desarrollo Tecnológico Positivo (Bers, 2008), ambos basados en el Construccionismo (Papert, 1993), además de los principios de educación inclusiva y el aprendizaje a través de juegos. Además, sigue los principios pedagógicos establecidos para la educación infantil del Decreto 183/2008 (Consejería de Educación y Universidades, 2008):

1. Actividad y experimentación: los niños y niñas captan la información a través de los sentidos, actuando, manipulando objetos físicos y experimentando.

2. Individualización: supone la adaptación a diferentes niveles de desarrollo de los niños y niñas, y a sus intereses, necesidades, posibilidades cognitivas, sociales, afectivas y motrices.

3. Enfoque globalizador: supone partir de lo concreto, conocido y experimentado por los niños y niñas, plantear actividades adaptadas a sus intereses y capacidades y conectar lo conocido con la nueva información permitiendo el progreso de cada niño/a según su propio ritmo.

\subsubsection{Movimiento "MAKER"}

El movimiento maker, iniciado hace una década, se ha extendido por todo el mundo con diferentes formatos, organizaciones y estructuras (hackerspaces, techshops, fablabs, espacios de talleres de clases, librerías, etc.) (Preddy, 2013; Hira, Hira y Hynes, 2014; Thompson, 2014). Los makerspaces se basan en una ideología constructivista integral para formar un enfoque construccionista de la educación (Kurti, Kurti y Fleming, 2014), siguiendo los principios de Piaget y Papert de aprender haciendo. Los principios constructivistas y construccionistas, a través de su énfasis en las oportunidades educativas activas, han llevado al desarrollo de la cultura creadora y enfoques enfocados en STEM para el aprendizaje y la participación de los estudiantes (Hamir, Maion, Tice y Wideman, 2015). Así, el conocimiento del aprendiz se construye creando e interactuando con objetos físicos. Por lo tanto, los espacios de construcción son una gran oportunidad para llevar la alfabetización mediática y las habilidades y competencias del siglo XXI a la gente (Ananiadou y Claro, 2010), como educación formal (en las escuelas) o informal (en las comunidades). Pero, el profesorado y las comunidades necesitan guías, herramientas y capacitación, y más investigación sobre cómo diseñar espacios para promover el aprendizaje basado en la realidad en diferentes contextos (escuelas, bibliotecas, comunidades, etc.) y los usuarios (niños/as, adolescentes y adultos).

Además, según los modelos construccionistas, los estudiantes aprenden mejor haciendo objetos tangibles. En este sentido, Papert argumentó que, debido a las propiedades particulares de los objetos físicos ofrecidos a los niños/as, limitan o mejoran lo que pueden construir, crear y aprender (Blikstein, 2018). La investigación ha demostrado que los objetos con affordances perceptuales y manipulativos afectan la imaginación de los niños/as pequeños (2-5 años de edad). Sin embargo, la mayoría de los estudios aceptan que "los objetos actúan como 
herramientas de desarrollo psicológico que tienen efectos inmediatos en los comportamientos de los niños/as" (Chu, Quek, Bhangaonkar, Ging y Sridharamurthy, 2015). De este modo, para que un objeto sea eficaz en el apoyo a la imaginación, debe permitir pasar "de la acción en respuesta a la percepción de los objetos a la acción generada y controlada por las ideas" (Chu et al., 2015).

Teniendo en cuenta los principios maker, se realizó una propuesta de makerspaces para la educación infantil que puede verse en (González y Aller, 2019).

\subsubsection{Desarrollo Tecnológico Positivo (PTD)}

En cuanto a la metodología que se propone seguir, se sustenta en los principios del Desarrollo Tecnológico Positivo (PTD) (Bers, 2008). El marco del PTD guía el desarrollo, la implementación y la evaluación de programas educativos que utilizan las nuevas tecnologías para promover el aprendizaje como un aspecto del desarrollo positivo de los niños y niñas. El marco pedagógico PTD es una extensión natural de la alfabetización informática y los movimientos tecnológicos que han influido en el mundo de la educación, añadiendo a los elementos cognitivos componentes psicosociales y éticos. Desde un punto de vista teórico, el marco pedagógico PTD se basa en un enfoque interdisciplinario que integra ideas de los campos de la comunicación mediada por ordenador, el aprendizaje colaborativo apoyado por ordenadores y la teoría construccionista del aprendizaje desarrollada por Seymour Papert (1993).

Como marco teórico, PTD propone seis comportamientos positivos (seis C) que deben ser apoyados por actividades educativas que utilizan nuevas tecnologías, como, por ejemplo, la robótica. Estos seis comportamientos son: la creación, la creatividad, la comunicación, la colaboración, la construcción de la comunidad y las opciones de conducta. Por tanto, siguiendo el marco PTD, en una actividad educativa basada en tecnología se propone que los niños y niñas desarrollen los siguientes comportamientos:

1. Creación de contenidos, mediante el diseño del robot y la programación de sus comportamientos. El proceso de diseño de ingeniería de la construcción y el pensamiento computacional involucrados en la programación fomentan la competencia en la alfabetización informática y la fluidez tecnológica. El uso de los "Diarios de Diseño de Ingeniería" (Figura 1) permite que los propios niños y niñas, así como para los docentes y la familia, puedan documentar su propio pensamiento, sus trayectorias de aprendizaje y la evolución del proyecto desarrollado con el robot KIBO en el tiempo.

2. Creatividad, realizando y programando proyectos personalmente significativos, resolviendo problemas de forma creativa y lúdica, integrando diferentes medios como robótica, motores, sensores, materiales reciclables, creando arte a través de un lenguaje de programación tangible con el robot.

3. Colaboración, involucrando a las niñas y niños en un ambiente de aprendizaje que promueva el trabajo en equipo, compartir recursos y preocuparse unos de otros mientras trabajan con sus robots. Al principio de cada jornada de trabajo, cada niño/a recibe, junto con su diario de diseño, una impresión personalizada con su fotografía en el centro de la página y las fotografías y nombres de todos los demás niño/as de la clase dispuestos en un círculo. A lo largo del día, con el mensaje del docente, cada niño/a dibuja una línea de su propia foto hacia las fotos de los niño/as con los que ha colaborado. La colaboración se define aquí como obtener ayuda o ayudar con un proyecto, programar juntos, prestar o pedir prestados materiales o trabajar juntos en una tarea común. Al final de la semana, los niño/as deben escribir o dibujan "tarjetas de agradecimiento" a los niño/as con los que han colaborado más.

4. Comunicación, a través de mecanismos que promuevan un sentido de conexión entre pares o con adultos. Por ejemplo, a través del uso de los círculos tecnológicos, cuando los niños/as dejan de trabajar, ponen sus proyectos sobre la mesa o el piso y comparten su proceso de aprendizaje. Los círculos tecnológicos representan una buena oportunidad para resolver problemas como comunidad. Algunos docentes invitan a todos los niños y niñas a sentarse juntos en un círculo para resolver problemas entre todos. También puede ser útil hacer un "Estacionamiento Robot" para que todos los robots puedan ir mientras no se están trabajando, de modo que los niños/as tengan las manos vacías y puedan concentrarse en los círculos tecnológicos. Cada aula tendrá sus propias rutinas y expectativas en torno a discusiones de 
grupo y tiempos dedicados al círculo, por lo que se anima a los docentes a adaptar lo que ya funciona en su clase para los círculos tecnológicos de esta propuesta didáctica.

5. Construcción de la comunidad, a través de promover la contribución de ideas en la comunidad de aprendizaje. Los proyectos finales realizados por los niños y niñas son compartidos con la comunidad a través de una demostración o una exposición abierta. Estas demostraciones o exposiciones brindan oportunidades para que los niños y niñas compartan y celebren el proceso y los productos tangibles de su aprendizaje con la familia y los amigos. A los niños y niñas se le da la oportunidad no sólo de ejecutar su robot, y al mismo tiempo, pueden desempeñar el papel de docente al explicar a su familia cómo lo construyeron, programaron y trabajaron a través de problemas.

6. Opciones de conducta, que proporcionan a los niños y niñas la oportunidad de experimentar con preguntas de "qué pasa si" y las consecuencias potenciales de cada opción. Estas preguntas permiten examinar los valores y explorar los rasgos de carácter de los niños y niñas al mismo tiempo que se trabaja con la robótica. El enfoque en aprender acerca de la robótica es tan importante como ayudar a los niños y niñas a desarrollar una brújula interior para guiar sus acciones de una manera justa y responsable. Una manera de alentar las opciones positivas es mediante el uso de "insignias de expertos/as". Los niños y niñas que dominan los conceptos rápidamente pueden ganar insignias expertas (una calcomanía para que se pongan). Un niño/a que lleva una insignia de experto/a utiliza el resto del período de clases ayudando a los estudiantes que tienen dificultades con los conceptos que han dominado.

\subsubsection{Educación inclusiva}

La educación es un derecho humano que debe ser asumido por las escuelas según la Declaración Mundial de los Derechos humanos, art. 26 (Naciones Unidas), y por tanto debe respetar la diversidad y asegurar que todas las personas tengan acceso a la misma. Desde este punto de vista, la UNESCO (2008), define a la educación inclusiva de la siguiente manera:

"La educación inclusiva puede ser concebida como un proceso que permite abordar y responder a la diversidad de las necesidades de todos los educandos a través de una mayor participación en el aprendizaje, las actividades culturales y comunitarias y reducir la exclusión dentro y fuera del sistema educativo. Lo anterior implica cambios y modificaciones de contenidos, enfoques, estructuras y estrategias basados en una visión común que abarca a todos los niños en edad escolar y la convicción de que es responsabilidad del sistema educativo regular educar a todos los niños y niñas. El objetivo de la inclusión es brindar respuestas apropiadas al amplio espectro de necesidades de aprendizaje tanto en entornos formales como no formales de la educación. La educación inclusiva, más que un tema marginal que trata sobre cómo integrar a ciertos estudiantes a la enseñanza convencional, representa una perspectiva que debe servir para analizar cómo transformar los sistemas educativos y otros entornos de aprendizaje, con el fin de responder a la diversidad de los estudiantes. El propósito de la educación inclusiva es permitir que los maestros y estudiantes se sientan cómodos ante la diversidad y la perciban no como un problema, sino como un desafío y una oportunidad para enriquecer las formas de enseñar y aprender".

La educación inclusiva puede ser vista como un principio y como derecho positivo, en donde se deben buscar las condiciones necesarias para que las personas puedan disfrutarlo efectivamente, eliminando las barreras y cualquier tipo de discriminación (Sarrionandía y Ainscow, 2011). Por tanto, nuestra propuesta educativa contempla como principio de educación inclusiva, atendiendo a la diversidad del alumnado y adaptando la práctica educativa a las características personales, intereses y necesidades de los niños y niñas. De esta forma, se contribuye a su desarrollo integral de los niños y niñas, dada la importancia que en estas edades adquieren el ritmo y el proceso de maduración. Nuestra propuesta promueve, además, la utilización de estrategias metodológicas para favorecer la atención a la diversidad (tutoría entre iguales, coevaluación, aprendizaje cooperativo, etc.). Asimismo, incluye principios de igualdad de género, promoviendo en las actividades la reflexión sobre roles y estereotipos en la ingeniería, y la utilización de lenguaje no sexista. 


\subsubsection{Aprendizaje a través de juegos}

Un elemento clave a tener en cuenta en la educación de los niños y niñas pequeños es el "aprendizaje a través del juego" o el "aprendizaje lúdico". Jugar es una de las formas más importantes en que los niños/as pequeños adquieren conocimientos y habilidades esenciales. Por esta razón, se deben brindar oportunidades y entornos que promuevan el juego, la exploración y el aprendizaje práctico para crear un programa educativo efectivo en la educación infantil (UNICEF, 2018). Algunas de las características de las experiencias lúdicas definen que al juego como (Zosh et al., 2017):

- Significativo: Los niños y niñas juegan para dar sentido al mundo que los rodea, y encuentran significado en la experiencia al conectarla con algo ya conocido. A través del juego, niños y niñas expresan y expanden la comprensión de sus experiencias.

- Alegría: Si observamos a los niños/a (o incluso a los adultos), jugando, podemos ver que a menudo sonríen y se divierten. Aunque el juego puede tener momentos de frustración y presentar retos a superar, en general las emociones que se despiertan son de disfrute, motivación y placer.

- Atracción: Si observamos a los niños/as jugando, podemos ver que se participan activamente, con compromiso, y combinan diferentes estados, tanto físico como mental.

- Iterativo: Los niños/a juegan para practicar habilidades, intentando y probando diferentes posibilidades, revisando hipótesis y descubriendo nuevos desafíos que conducen a un aprendizaje más profundo.

- Interacción social: El juego les permite a los niños/as comunicar ideas, les ayuda a entender a los demás a través de interacción social, construyendo relaciones más fuertes a través de una mejor comprensión de las mismas.

Un aspecto importante del juego es el control que tienen los niños/as sobre la experiencia, en cuanto a su iniciativa, toma de decisiones y elecciones personales sobre el juego. Por otra parte, los niños/as aprenden habilidades críticas para su desarrollo mientras juegan, sin pensar que su objetivo es aprender algo. El juego crea poderosas oportunidades de aprendizaje en todas las áreas de desarrollo infantil, incluidos los motores, cognitivos y las habilidades sociales y emocionales (Zosh et al., 2017).

Los niños y niñas son aprendices "prácticos": adquieren conocimiento a través de la interacción lúdica con objetos y personas (Resnick y Robinson, 2017). Necesitan mucha práctica con objetos físicos para comprender conceptos abstractos. Por ejemplo, jugando con bloques geométricos entienden el concepto de que dos cuadrados pueden formar un rectángulo y dos triángulos pueden formar un cuadrado o en un baile, pueden aprender un patrón como dar un paso adelante, dar un paso atrás girar, aplaudir y repetir, y comienzan a comprender las características de los patrones, que son la base de las matemáticas. Asimismo, el juego simbólico es muy beneficioso, los niños/as pueden expresar sus ideas, pensamientos y sentimientos, aprenden a controlar sus emociones, interactuar con otros, resolver conflictos y adquirir un sentido de competencia.

Por otra parte, el juego sienta las bases para el desarrollo del pensamiento crítico, social y emocional. A través del juego, los niños aprenden a forjar conexiones con otros y a compartir y negociar. En general, el juego es una forma de expresión humana, y desarrolla la imaginación, la curiosidad y la creatividad, que son competencias clave para el siglo XXI (Honey y Kanter, 2013).

Por todo lo anterior, nuestra propuesta inclusiva de enseñanza-aprendizaje de pensamiento computacional y la programación para la educación infantil se sustenta en los principios del aprendizaje a través del juego.

\subsection{Propuesta}

Esta propuesta educativa inclusiva para la enseñanza-aprendizaje de la programación y el pensamiento computacional para la educación infantil, basada en los principios anteriormente 
descritos, introduce "ideas poderosas" sobre éstos contenidos. Según Papert (1993), las ideas poderosas ofrecen nuevas formas de pensar, nuevas formas de poner en práctica el conocimiento y nuevas formas de hacer conexiones personales y epistemológicas con otros dominios de conocimiento (Bers, 2018, p.70). En este caso, las ideas poderosas a trabajar incluyen: el proceso de diseño de ingeniería, la robótica, la programación y los sensores (Tabla 1). Asimismo, se presentan de forma estructurada el conjunto de actividades a realizar (Figura 1).

Tabla 1. Ideas poderosas a desarrollar

\begin{tabular}{|c|c|c|}
\hline $\begin{array}{c}\text { Idea } \\
\text { poderosa }\end{array}$ & Descripción & Objetivos \\
\hline Idea 1 & $\begin{array}{l}\text { Proceso de diseño de } \\
\text { ingeniería }\end{array}$ & $\begin{array}{l}\text { - Comprender que la gente tiene que } \\
\text { planificar para hacer las cosas } \\
\text { - Comprender que todas las personas } \\
\text { pueden diseñar soluciones a un } \\
\text { problema. } \\
\text { - Comprender que el diseño es un } \\
\text { proceso creativo (que conduce a } \\
\text { productos y sistemas útiles). } \\
\text { - Comprender que todos los diseños } \\
\text { pueden ser mejorados. } \\
\text { - Comprender que el proceso de diseño } \\
\text { de ingeniería incluye identificar un } \\
\text { problema, buscar ideas, desarrollar } \\
\text { soluciones y compartir soluciones con } \\
\text { otros. } \\
\text { - Hacer preguntas y hacer } \\
\text { observaciones ayuda a una persona a } \\
\text { averiguar cómo funcionan las cosas. } \\
\text { - Solucionar problemas es una manera } \\
\text { de averiguar por qué algo no funciona } \\
\text { para que pueda arreglarse. }\end{array}$ \\
\hline Idea 2 & Robótica & $\begin{array}{l}\text {-Construir un objeto utilizando el } \\
\text { proceso de diseño. } \\
\text {-Descubrir cómo funcionan las cosas. } \\
\text {-Comprender que los sistemas tienen } \\
\text { partes que trabajan juntas para lograr } \\
\text { un objetivo. } \\
\text {-Comprender que las herramientas, las } \\
\text { máquinas, etc. utilizan la energía para } \\
\text { poder hacer su trabajo. }\end{array}$ \\
\hline Idea 3 & Programación & $\begin{array}{l}\text { - Reconocer y usar símbolos cotidianos } \\
\text { - Comprender que las personas usan } \\
\text { símbolos cuando se comunican a través } \\
\text { de la tecnología. } \\
\text { - Comprender que el estudio de la } \\
\text { tecnología utiliza muchas de las mismas } \\
\text { ideas y habilidades que otros temas. }\end{array}$ \\
\hline Idea 4 & Sensores & $\begin{array}{l}\text {-Comprender que el mundo natural y el } \\
\text { mundo humano son diferentes. }\end{array}$ \\
\hline
\end{tabular}




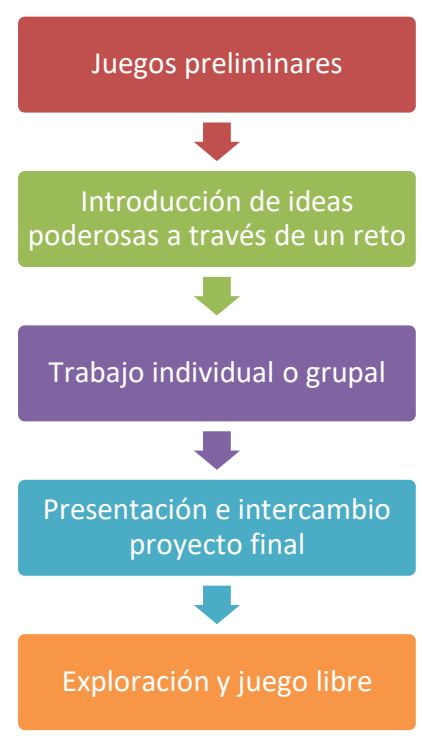

Figura 1. Estructura de la secuencia de actividades a realizar en las sesiones.

Por otra parte, además de los objetivos de aprendizajes que los niños y niñas relacionados con las ideas poderosas, pueden aprender otros contenidos del currículum forma transversal y conectar la programación y la resolución de problemas con el contexto.

En cuanto al pensamiento computacional (PC), las competencias que deben desarrollar son las siguientes (Bers, 2018, p.57): a) hardware / software (los objetos inteligentes no son mágicos, los objetos están diseñados por humanos); b) algoritmos (secuenciación / orden, organización lógica); c) modularidad (división de tareas más grandes en partes más pequeñas, instrucciones), estructuras de control (reconocimiento de patrones y repetición, causa y efecto); d) representación (representación simbólica, modelos); e) proceso de diseño (resolución de problemas, perseverancia, edición / revisión) y f) depuración (identificación de problemas, resolución de problemas, perseverancia).

\subsubsection{Gestión del aula}

La enseñanza de la robótica y de la programación en un aula para la primera infancia requiere una planificación cuidadosa y realizar ajustes para lograr una buena gestión de aula. Las actividades de robótica, debido a la novedad y el comportamiento de los materiales en sí, requerirán de una atención diferente. Se recomienda seguir una estructura de espacio "maker" para la educación infantil. Los docentes deben encontrar lo que funciona en sus circunstancias particulares. En general, se debe proporcionar y enseñar a los niños y niñas una estructura clara y conjunto de normas para el uso de materiales y para las rutinas de cada parte de las actividades y asegurarse de que los estudiantes comprendan los objetivos de cada actividad. Los carteles y las ayudas visuales pueden facilitar a los niños y niñas las respuestas a sus propias preguntas y recordar nueva información. De los diferentes espacios en el aula, podemos utilizar algunos de los rincones típicos (tales como: el rincón del encuentro y biblioteca, el rincón de construcciones para la construcción del robot, el rincón de plástica para la decoración del robot, etc.

\subsubsection{Trabajo individual y grupal}

El trabajo individual con los robots depende de la disponibilidad de los recursos, que, en la mayoría de los casos, suelen ser limitados. Sin embargo, se puede organizar el trabajo en pequeños grupos o también para algunas actividades, en el grupo de clase completo.

\subsubsection{Recursos}

Los materiales que se proponen utilizar son robots tangibles manipulativos sin necesidad de conexión (desenchufado) y materiales plástica y de reciclaje. Los materiales que nuestro alumnado explora y manipula tienen una importancia decisiva en sus procesos de aprendizaje. Por ello, se deben seleccionar, organizar y distribuir los mismos de forma que promuevan la autonomía, la relación-comunicación con sus pares (puesto que necesitan de los demás para 
jugar, para realizar tareas en equipo, para comunicar sus descubrimientos), el desarrollo afectivo, el desarrollo motriz, el desarrollo de los lenguajes expresivos, el conocimiento físico de los objetos (al explorar y descubrir sus cualidades y establecer relaciones entre ellos), el contacto con materiales estructurados (puzles, bloques lógicos, construcciones, etc.) y el contacto con materiales no estructurados (agua, arena, piedras, semillas y otros materiales procedentes de la naturaleza y materiales de reciclaje).

Los proyectos de robótica de aula requieren una gran cantidad de piezas y materiales, y la cuestión de cómo manejarlos trae a colación varias cuestiones clave que pueden apoyar o dificultar el éxito de su desarrollo. La primera cuestión es la accesibilidad de los materiales. Algunos docentes pueden optar por dar un kit completo de materiales para cada niño/a, para una pareja o para una mesa con varios niños y niñas. Otros docentes pueden optar por desmontar los kits y ordenar los materiales por tipo y colocar todos los materiales en una ubicación central. Dado que diferentes proyectos requieren diferentes elementos de robótica y programación, esta configuración puede permitir que los niños y niñas tomen sólo lo que necesitan y dejan otras partes para los otros niños y niñas que los necesitan.

\subsubsection{Evaluación}

Es importante evaluar el proceso de aprendizaje del estudiante y los resultados de dicho aprendizaje. Esto se puede hacer documentando los proyectos de los estudiantes y las maneras de hablar y compartir sus proyectos con el resto de la clase. Evaluar el aprendizaje individual de los niños y niñas mientras trabajan en grupos, puede ser un desafío. Los criterios de evaluación de cada actividad se pueden organizar en una secuencia de logros concretos que conducen a una insignia relacionada con su nivel de habilidad. Los niños y niñas explorarán y aprenderán a diferentes ritmos. Por ello, el docente deberá ir regulando el ritmo de la clase, de forma que todos lleguen a obtener la insignia correspondiente en cada fase. Asimismo, se sugiere utilizar rúbricas para evaluar los diferentes niveles de competencia adquirida y llevar diarios de clase. Se podrá hacer uso además de herramientas específicas de evaluación de las habilidades de programación y de la lista de verificación de comportamientos positivos de desarrollados por la tecnología, además de otros instrumentos específicos relacionados a la evaluación emocional.

\section{CONCLUSIONES}

En este artículo se ha presentado un conjunto de principios y estrategias de enseñanzaaprendizaje de la programación y el pensamiento computacional en la educación infantil con un enfoque inclusivo. Los principales principios en los que se basa la propuesta son los siguientes: movimiento maker, desarrollo positivo de la tecnología (PTD), educación inclusiva y aprendizaje a través de juegos. Además, se siguen los principios pedagógicos establecidos para la educación infantil de actividad y experimentación, individualización y enfoque globalizador. De acuerdo a la revisión bibliográfica presentada en el trabajo, éstos principios siguen los enfoques constructivistas y construccionistas para la enseñanza de la programación y el pensamiento computacional, además de estar de acuerdo a considerar a éstas nuevas competencias y habilidades como una nueva alfabetización, necesaria para todas las personas en la sociedad actual y futura.

En cuanto a la propuesta realizada se propone la enseñanza a través de "ideas poderosas" relacionadas con la informática, tales como el proceso de diseño de ingeniería, la robótica, la programación y los sensores. Estas ideas se relacionan con objetivos de aprendizaje específicos y se propone además su conexión con otras competencias de pensamiento computacional y con su relación con otros contenidos curriculares y competencias transversales. Asimismo, se proponen estrategias para la estructura de las actividades, la gestión de recursos y del aula, el trabajo individual y grupal y la evaluación.

Aunque la propuesta presentada en éste trabajo ha sido validada previamente con niños y niñas y sus educadores en diferentes centros educativos, así como en contextos especiales como aulas hospitalarias o con personas con síndrome de Down, mostrando resultados positivos, como limitaciones de la propuesta presentada, debemos mencionar que se debe ampliar la validación de la misma a otros contextos y entornos, así como con otros colectivos que presentan necesidades educativas y específicas. Además, como posibles mejoras de la propuesta se deben 
incluir guías de actividades y secuencias didácticas que faciliten a los docentes la incorporación de la enseñanza del pensamiento computacional y la programación en sus aulas.

\section{REFERENCIAS BIBLIOGRÁFICAS}

Ananiadou, K. y Claro, M. (2010). Habilidades y competencias del siglo XXI para los aprendices del nuevo milenio en los países de la OCDE. Organización para la Cooperación y el Desarrollo Económico. Recuperado de: shorturl.at/cnrDP.

Bers, M. U. (2008). Blocks, robots and computers: Learning about technology in early childhood. Teacher's College Press, NY.

Bers, M. U., González-González, C., y Armas-Torres, M. B. (2019). Coding as a playground: Promoting positive learning experiences in childhood classrooms. Computers \& Education, 138, 130-145. doi: https://doi.org/10.1016/i.compedu.2019.04.013

Bers, M.U. (2010). Beyond computer literacy: Supporting youth's positive development through technology. New Directions for Youth Development, 128, 13-23. doi: https://doi.org/10.1002/yd.371

Bers, M.U. (2017). The Seymour test: Powerful ideas in early childhood education, International Journal of Child-Computer Interaction, 14,10-14. doi: http://dx.doi.org/10.1016/j.ijcci.2017.06.004.

Bers, M. U. (2018). Coding as a playground: Programming and computational thinking in the early childhood classroom. Routledge.

Blikstein, P. (2018). Maker Movement in Education: History and Prospects. Handbook of Technology Education, 419-437. doi: https://doi.org/10.1007/978-3-319-44687-5 33

Burlson, W., Harlow, D. B., Nilsen, K. J., Perlin, K., Freed, N., Jensen, C., Lahey, B., Lu, P., y Muldner, K. (2017). Active Learning Environments with Robotic Tangibles: Children's Physical and Virtual Spatial Programming Experiences. IEEE Transactions on Learning Technologies. doi: https://doi.org/10.1109/TLT.2017.2724031

Chen, G., Shen, J., Barth-Cohen, L., Jiang, S., Huang, X., y Eltoukhy, M. (2017). Assessing elementary students' computational thinking in everyday reasoning and robotics programming. Computers \& Education, 109, 162-175. doi: https://doi.org/10.1016/j.compedu.2017.03.001

Ching, Y. H., Hsu, Y. C., y Baldwin, S. (2018). Developing Computational Thinking with Educational Technologies for Young Learners. TechTrends, 62, 563-573. doi: https://doi.org/10.1007/s11528-018-0292-7

Chu, S. L., Quek, F., Bhangaonkar, S., Ging, A. B., y Sridharamurthy, K. (2015). Making the Maker: A Means-to-an-Ends approach to nurturing the Maker mindset in elementary-aged children. International Journal of Child-Computer Interaction, 5,11-19. doi: https://doi.org/10.1016/j.ijcci.2015.08.002

Cunha, F., y Heckman, J. (2007). The technology of skill formation. American Economic Review, 97(2), 31-47.doi: https://doi.org/10.3386/w12840.

Consejería de Educación y Universidades (2008). Decreto 183/2008, de 29 de julio, por el que se establece la ordenación y el currículo del $2^{\circ}$ ciclo de la Educación Infantil en la Comunidad Autónoma de Canarias. Recuperado de: shorturl.at/bkmDY

Di Lieto, M. C., Inguaggiato, E., Castro, E., Cecchi, F., Cioni, G., Dell'Omo, M., Laschi, C., Pecini, C., Santerini, G., Sgandurra, G. y Dario, P. (2017). Educational Robotics intervention on 
Executive Functions in preschool children: A pilot study. Computers in human behavior, 71, 16-23. doi: https://doi.org/10.1016/j.chb.2017.01.018

García-Peñalvo, F. J., y Mendes, A. J. (2018). Exploring the computational thinking effects in preuniversity education. Computers in Human Behavior, 80, 407-411. doi: https://doi.org/10.1016/j.chb.2017.12.005.

González-González, C. S., y Arias, L. G. A. (2019). Maker movement in education: maker mindset and makerspaces. En Libro "Ingeniería colaborativa, aplicaciones y usos desde la perspectiva de la Interacción Humano-Computador". Jurado, J.L., Collazos, C.A. y Muñoz, L. F. (Eds.). Editorial: Universidad San Buenaventura de Cali. Colombia. (p. 297-307).

González-González, C. S., Cáceres-García, L., y Violant-Holz, V. (2019). Bringing Computational Thinking to Hospital Classrooms. In Proceedings of the Seventh International Conference on Technological Ecosystems for Enhancing Multiculturality (31-35). ACM. doi: https://doi.org/10.1145/3362789.3362908.

González-González, C. S., Herrera-González, E., Moreno-Ruiz, L., Reyes-Alonso, N., Hernández-Morales, S., Guzmán-Franco, M. D., y Infante-Moro, A. (2019). Computational Thinking and Down Syndrome: An Exploratory Study Using the KIBO Robot. Informatics, 6 (2), 25. doi: https://doi.org/10.3390/informatics6020025

Chen, G., Shen, J., Barth-Cohen, L., Jiang, S., Huang, X., y Eltoukhy, M. (2017). Assessing elementary students' computational thinking in everyday reasoning and robotics programming. Computers \& Education, 109, 162-175. doi: https://doi.org/10.1016/i.compedu.2017.03.001

Halverson, E. R., y Sheridan, K. (2014). The maker movement in education. Harvard educational review, 84(4), 495-504. doi: https://doi.org/10.17763/haer.84.4.34j1g68140382063

Hamir, S., Maion, S., Tice, S., y Wideman, A. (2015). ETEC 512. Constructivism in Education. Recuperado de shorturl.at/emuwG

Hira, A., Hira, J., y Hynes, M. M. (2014). Classroom makerspaces: Identifying the opportunities and challenges. In 2014 IEEE Frontiers in Education Conference (FIE) Proceedings (1-5). IEEE. doi: 10.1109/FIE.2014.7044263

Honey, M., y Kanter, D. E. (2013). Design, make, play: Growing the next generation of STEM innovators. Routledge.

Naciones Unidas (2015). Declaración Mundial de los Derechos Humanos. Recuperado de: shorturl.at/lmxNO.

Jung, S., y Won, E. (2018). Systematic Review of Research Trends in Robotics Education for Young Children. Sustainability, 10(4), 905.doi: https://doi.org/10.3390/su10040905

Kandlhofer, M., y Steinbauer, G. (2016). Evaluating the impact of educational robotics on pupils' technical-and social-skills and science related attitudes. Robotics and Autonomous Systems, 75, 679-685. doi: https://doi.org/10.1016/j.robot.2015.09.007

Kurti, R., Kurti, D., y Fleming, L. (2014). Practical implementation of an educational makerspace. Teacher Librarian, 42(2), 20. Recuperado de: shorturl.at/cdpuF

Madill, H. M., Campbell, R. G., Cullen, D. M., Armour, M. A., Einsiedel, A. A., Ciccocioppo, A. L., y Rothwell, C. J. (2007). Developing career commitment in STEM-related fields: myth versus reality. Women and Minorities in Science, Technology, Engineering and Mathematics, 210. doi: https://doi.org/10.4337/9781847206879 
Manches, A., y Plowman, L. (2015). Computing education in children's early years: A call for debate. British Journal of Educational Technology, 48(1), 191-201. doi: https://doi.org/10.1111/bjet.12355.

Metz, S. S. (2007). Attracting the engineers of 2020 today. Women and minorities in science, technology, engineering, and mathematics: Upping the numbers, 184-209.

Öztürk, H. T., y Calingasan, L. (2018). Robotics in Early Childhood Education: A Case Study for the Best Practices. In H. Ozcinar, G. Wong, y H. Ozturk (Eds.). Teaching Computational Thinking in Primary Education (182-200). Hershey, IGI Global. doi: https://doi.org/10.4018/978-1-5225-3200-2.ch010.

Papert, S. (1993). The children's machine: Rethinking school in the age of the computer. BasicBooks, 10 East 53rd St., New York, NY 10022-5299.

Preddy, L. (2013). Creating school library "makerspace". School Library Monthly, 29(5), 41-42. Recuperado de: shorturl.at/bnQT7

Resnick, M. y Robinson, K. (2017). Lifelong kindergarten: Cultivating creativity through projects, passion, peers, and play. MIT press.

Román-González, M., Moreno-León, J., y Robles, G. (2019). Combining Assessment Tools for a Comprehensive Evaluation of Computational Thinking Interventions. In Computational Thinking Education (79-98). Springer, Singapore. doi: https://doi.org/10.1007/978-981-13$\underline{6528-7 \quad 6}$

Sarrionandía, G. E. y Ainscow, M. (2011). La educación inclusiva como derecho. Marco de referencia y pautas de acción para el desarrollo de una revolución pendiente. Tejuelo: didáctica de la lengua y la literatura. Educación, 12, 26-46. Recuperado de: shorturl.at/adoQT

Serholt, S. (2018). Breakdowns in children's interactions with a robotic tutor: A longitudinal study. Computers in Human Behavior, 81, 250-264. doi: https://doi.org/10.1016/j.chb.2017.12.030

Siu-Cheung, K. (2019). Components and Methods of Evaluating Computational Thinking for Fostering Creative Problem-Solvers in Senior Primary School Education. Computational thinking Education. NY: Springer Berlin Heidelberg, 2019. doi:https://doi.org/10.1007/978-981$\underline{13-6528-7 \quad 8}$

Steele, C. M. (1998). Stereotyping and its threat are real. American Psychologist, 53, 680-681.

Thompson, G. (2014). The maker movement connects to the classroom. The Education Digest, 80(3), 34. Recuperado de: shorturl.at/bitKQ

UNESCO (2008). Educación Inclusiva. Recuperado de: shorturl.at/gmFX8

UNICEF (2018). Learning through play: Strengthening learning through play in early childhood education programmes. New York: UNICEF. Recuperado de: shorturl.at/fhjmU

Zosh, J. N., Hopkins, E. J., Jensen, H., Liu, C., Neale, D., Hirsh-Pasek, K., Solis, S., y Whitebread, D. (2017). Learning through play: a review of the evidence. LEGO Fonden. Recuperado de: shorturl.at/jICT8 


\section{INFORMACIÓN SOBRE LA AUTORA}

\section{Carina Soledad González González Universidad de La Laguna}

Profesora Catedrática de la Universidad de La Laguna (España) en el Departamento de Ingeniería Informática y de Sistemas. Sus áreas de especialización son la Informática Educativa y la Interacción Persona-Ordenador. Desde hace más de 20 años ha centrado su especialización en el campo de la Informática Educativa y la Interacción Persona-Ordenador (Sistemas Tutoriales Inteligentes, Interfaces adaptativas y personalizables, Videojuegos Educativos, Gamificación, elearning, cultura digital). Ha publicado más de un centenar de artículos de investigación sobre esta temática.

Los textos publicados en esta revista están sujetos a una licencia de Reconocimiento 4.0 España de Creative Commons. Puede copiarlos, distribuirlos, comunicarlos públicamente y hacer obras derivadas siempre que reconozca los créditos de las obras (autoría, nombre de la revista, institución editora) de la manera especificada por los autores o por la revista. La licencia completa se puede consultar en:Licencia Creative Commons Atribución-NoComercial-Compartir por igual 4.0 Internacional.
} 